\title{
Análisis comparativo de los casos confirmados y defunciones por Covid-19 en la población de tres zonas metropolitanas de México
}

\section{Comparative analysis of confirmed cases and deaths from Covid-19 in the population of three metropolitan areas of Mexico}

\author{
Daniel Lozano-Keymolen, Sergio Cuauhtémoc Gaxiola-Robles Linares \\ y Bernardino Jaciel Montoya-Arce
}

\author{
Centro de Investigación y Estudios Avanzados de la Población \\ de la Universidad Autónoma del Estado de México, México
}

\begin{abstract}
Resumen
La enfermedad infecciosa denominada Covid-19 causada por el coronavirus SARS-CoV-2 es una de las pandemias que la humanidad enfrenta. Por sus consecuencias sociales, económicas y en la salud, la Covid-19 ha mostrado los efectos de la explotación animal y la invasión humana a entornos reservorios de patógenos desconocidos que derivaron en una enfermedad mortal para el ser humano. En particular, las zonas metropolitanas han sido espacios con grandes afectaciones de la Covid-19 por las concentraciones poblacionales que derivan en un mayor número de contagios. En este contexto, el presente artículo tiene como objetivo realizar un análisis comparativo de los casos confirmados, las defunciones y las prevalencias de enfermedades en infección por Covid-19 para la Zona Metropolitana de Monterrey, la zona metropolitana de Guadalajara y la Zona Metropolitana del Valle de México en dos periodos. La fuente de información que se utilizó fueron los datos de la Secretaría de Salud de México y la Encuesta Intercensal. La metodología consistió en dos etapas que permitieron realizar el análisis comparativo de las tres zonas metropolitanas.
\end{abstract}

Palabras clave: Covid-19, diabetes, hipertensión, obesidad, zonas metropolitanas, México.

Abstract

The infectious disease called Covid-19 caused by the SARS-CoV-2 coronavirus is one of the pandemics that humanity faces. Due to its social, economic and health consequences, Covid-19 has shown the effects of animal exploitation and human invasion to reservoir environments for unknown pathogens that resulted in a fatal disease for humans. In particular, metropolitan areas have been areas with great effects of Covid-19 due to population concentrations that lead to a greater number of infections. In this context, this article aims to carry out a comparative analysis of confirmed cases, deaths, and prevalences of diseases in Covid-19 infection for the Monterrey metropolitan area, the Guadalajara metropolitan area, and the Valle Metropolitan Area. of Mexico in two periods. The source of information used was data from the Mexican Ministry of Health and the Intercensal Survey. The methodology consisted of two stages that allowed for the comparative analysis of the three metropolitan areas.

Keywords: Covid-19, diabetes, hypertension, obesity, metropolitan areas, Mexico. 


\section{INTRODUCCIÓN}

\section{La pandemia de la Covid-19}

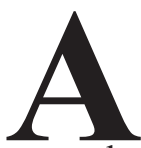

lo largo de la historia de la humanidad, las pandemias han tenido impactos devastadores en la población mundial. Los principales efectos se han concentrado en la morbilidad y la mortalidad provocando una serie cambios en la dinámica, la estructura y la composición de la población. En este sentido, la demografía es una disciplina que ha coadyuvado a conocer el comportamiento de las pandemias ${ }^{1}$ a través del tiempo (Kalabikhina, 2020).

Las pandemias con mayor impacto en la mortalidad de los seres humanos han sido la viruela, el sarampión, la gripe española, la peste negra y la ocasionada por el Virus de Inmunodeficiencia Humana (VIH). En particular, se estima que la viruela ${ }^{2}$ ocasionó 300 millones de muertes entre la población mundial (Castañeda y Ramos, 2020). La viruela se desarrolló hace miles de años y fue hasta el siglo XVIII que se encontró la primera vacuna que logró reducir la mortalidad (Larrea, 2007). Aunque desde 1980, la Organización Mundial de la Salud (OMS) declaró como erradicada la enfermedad, existen laboratorios que guardan cepas, lo cual constituye un importante factor de riesgo para la salud de la población mundial ya que desde la década de los años setenta del siglo XX no se aplica la vacuna (Moreno-Sánchez et al., 2018).

En la actualidad, la humanidad se enfrenta a una nueva pandemia denominada Covid-19 y que es causada por el coronavirus SARS-CoV-2. Esta pandemia tuvo su origen en la ciudad de Wuhan, China, que oficialmente reportó la aparición de diversos conglomerados de una neumonía de origen desconocido en diciembre de 2019. Diversos estudios muestran que la Covid-19 es una enfermedad que se desarrolló de forma natural mediante patógenos que pasan de animales a humanos, este coronavirus es altamente contagioso y se transmite de persona a persona (Zhu et al., 2020). La OMS declaró el 11 de marzo de 2020 como pandemia el brote de Covid-19 dado el número de casos reportados, así como la diversidad de territorios con reportes de casos confirmados (OMS, 2020).

1 De acuerdo con Shoals (2018: 6-7), “una epidemia es el brote de una enfermedad que se propaga raṕidamente afectando a muchas personas... Una pandemia es una enfermedad infecciosa que afecta a grandes poblaciones cuando una epidemia se propaga a través de sus límites geográficos y comienza a afectar las regiones cercanas. Todas las pandemias son epidemias, pero no todas las epidemias pueden llamarse pandemias porque se limitan a un área en particular".

${ }_{2}^{2}$ La viruela es la infección causada por el poxvirus vareola que se transmite por contacto directo con la saliva, la piel o la ropa de quienes padecen la enfermedad (Moreno-Sánchez et al., 2018). 
Una vez que se declara como pandemia, los gobiernos de diversos países activan una serie de protocolos para intentar disminuir la transmisión de la enfermedad, sin embargo, los contagios por la Covid-19 se incrementaron en todas las regiones. En particular, las muertes en mayor cuantía por este coronavirus se presentaron en la población adulta mayor, así como entre aquellos individuos con antecedentes de alguna enfermedad crónica como diabetes, hipertensión arterial o enfermedad cardiovascular (Ejaz et al., 2020; Mueller et al., 2020).

\section{Situación Internacional de la Covid-19}

En el año 2020, el principal reto que enfrenta la humanidad es la enfermedad infecciosa de la Covid-19 cuya presencia se da en prácticamente todos los continentes. Según la OMS, hasta el 14 de enero de 2021 se acumulan poco más de 90 millones de casos confirmados y cerca de 2 millones de muertes por Covid-19 a nivel mundial. El impacto de esta enfermedad ha sido diferenciado en los países, sin embargo, una de las regiones con mayor casos confirmados y muertos por Covid-19 es el continente Americano con más del 44 por ciento de los casos confirmados en el mundo (OMS, 2021).

En este sentido, los países de América han sufrido de manera importante los estragos de esta pandemia, por ejemplo, los Estados Unidos de América hasta el 15 de diciembre del2020 tenían cerca de 19 millones de casos y aproximadamente 300 mil muertes. Para la región de Latinoamérica, los casos de Covid-19 en la misma fecha alcanzaban 12 millones de casos y cerca de 400 mil muertes (véase Tabla 1). Lo anterior expone que América Latina es una de las regiones más vulnerables por la falta de infraestructura hospitalaria adecuada, las carencias o deficiencias de los sistemas de atención a la salud o por poblaciones con altas prevalencias de enfermedades crónicas como diabetes u obesidad que agravan la Covid-19 (Burki, 2020).

Como se puede observar en la Tabla 1 y a la fecha de corte, en Latinoamérica, el país con mayor número de casos confirmados por cada 100 mil habitantes era Argentina con 3,394, el segundo país más afectado es Perú con poco más de tres mil casos por cada 100 mil personas, mientras que en el caso de México había cerca de 1,000 casos confirmados por cada 100 mil individuos. En las defunciones, el comportamiento es similar a lo observado para los casos confirmados para estos países. Por ejemplo, en México había 89 defunciones por cada 100 mil habitantes, aunque Perú mostraba una cifra superior al ser de 115 muertes. 
Tabla 1: Número casos y defunciones por 100 mil habitantes según países seleccionados de América Latina, 15 de diciembre 2020

\begin{tabular}{lrrrr}
\hline & \multicolumn{2}{c}{ Casos confirmados } & \multicolumn{2}{c}{ Defunciones } \\
País & Totales & $\begin{array}{r}\text { Por 100 mil } \\
\text { habitantes }\end{array}$ & Totales & $\begin{array}{r}\text { Por 100 mil } \\
\text { habitantes }\end{array}$ \\
\hline Argentina & $1^{\prime} 510,203$ & 3,394 & 41,204 & 93 \\
Brasil & 7'040,608 & 3,353 & 183,735 & 87 \\
Chile & 575,329 & 3,028 & 15,949 & 84 \\
Colombia & $1 ' 444,646$ & 2,872 & 39,356 & 78 \\
Ecuador & 202,356 & 1,190 & 13,896 & 82 \\
México & $1 ' 267,202$ & 975 & 115,099 & 89 \\
Perú & 986,130 & 3,082 & 36,754 & 155 \\
\hline
\end{tabular}

Fuente: elaboración propia con base en https://coronavirus.jhu.edu/map.html

Entonces, los efectos en los países latinoamericanos han sido devastadores (Ramos, 2020). En particular, la región latinoamericana ha sido afectada en sus sistemas de salud y en los aspectos económicos, debido a que se pronostica una contracción de casi nueve por ciento en la región, lo que implica impactos en la población más vulnerable, así como en el mercado laboral debido a los cambios generados por la pandemia de la Covid-19 (CEPAL, 2020).

Aunado a lo anterior, un elemento relevante para la rápida propagación del coronavirus es la concentración de la población en mega ciudades. Los países latinoamericanos se caracterizan por contar con grandes aglomeraciones de la población, por ejemplo en 2018, la Zona Metropolitana del Valle de México contaba con casi 21.5 millones de personas, en lo que respecta a Sao Paulo en Brasil contaba con aproximadamente 21.6 millones y Gran Buenos Aires de Argentina eran 12.4 millones de personas (ONU, 2018).

\section{Urbanización, zonas metropolitanas y Covid-19}

El término urbanización implica diversas connotaciones sobre los factores que han impulsado este tipo de cambio a nivel mundial. Procesos demográficos como la migración o las diferencias en la mortalidad y la fecundidad entre el campo y la ciudad suelen relacionarse como factores impulsores de los procesos de urbanización, sin embargo, el término urbanización puede emplearse para referirse al cambio de una población menos densamente 
poblada a una más poblada (Reyes et al., 2013). Entonces, esta es la definición que se asume en este trabajo.

Los procesos de urbanización y la formación de zonas metropolitanas se han propuesto como un gran suceso en las sociedades modernas. Sin embargo, la urbanización experimentada a lo largo del último siglo ha mostrado factores negativos como el abandono de las zonas rurales, el hacinamiento, el aumento de la pobreza y la desigualdad, deficiencia o ausencia de servicios de agua potable, saneamiento y limpieza, que constituyen elementos que incentivan la transmisión de enfermedades e infecciones (Reyes et al., 2013). Esto es particular para el caso de las naciones en desarrollo y las menos desarrolladas en las que se reafirman las desigualdades sociales y económicas de estas poblaciones, pues los procesos de urbanización han sido acelerados disminuyendo la posibilidad de mejorar las condiciones de habitabilidad para las personas (Capolongo et al., 2020; Lee et al., 2020).

Sin embargo, es notorio que la actual pandemia de Covid-19 no está exclusivamente relacionada con las condiciones sociales, económicas y de recursos en salud de los países de economías en desarrollo y las menos desarrolladas, pues las economías desarrolladas experimentan la pandemia con efectos casi tan catastróficos como los de los primeros países. Al respecto, y sobre la velocidad de transmisión de la Covid-19 a escala global, se ha discutido sobre la importancia de factores como la elevada interacción humana impulsada por los medios de transporte (Iacus et al., 2020).

Entre los mecanismos que explican gran parte de los cambios en las causas de muerte a lo largo de la historia se encuentran el hambre, la violencia y las enfermedades. Particularmente, las enfermedades infecciosas se caracterizan porque sus mecanismos básicos de propagación son los fluidos corporales de un individuo infectado a otro no infectado. De manera tal que el contacto entre personas aumenta la probabilidad de transmisión de infecciones y la urbanización se ha señalado como un factor que incrementa la posibilidad de contacto y exposición entre los individuos a las enfermedades infecciosas (Reyes et al., 2013).

Aunado a lo anterior, la rápida urbanización incentivada por el desarro1lo económico que condujo a los procesos de expansión urbana, que entre otras cosas, provocan la invasión de hábitats naturales, intervienen en los corredores ecológicos y los ecosistemas o aumentar el contacto entre las personas y los animales silvestres, incrementan las posibilidades para las infecciones zoonóticas. Esto es importante dado que, entre 1940 y 2004 se identificaron 335 enfermedades infecciosas emergentes de las cuales más 
de 60 por ciento eran de origen zoonótico como en el caso de la Covid-19 (Capolongo et al., 2020: 14).

La Covid-19 es una enfermedad que muestra parte de las complejidades que las sociedades enfrentan, sobre todo aquellas con fuertes niveles de urbanización dado que este virus ha modificado las formas en las que las personas, las familias y las comunidades conviven y laboran (Capolongo et al., 2020; Sharifi y Khavarian-Garmsir, 2020). De manera tal que, la presencia de elevadas concentraciones poblacionales como las características de las zonas urbanas y metropolitanas exponen las carencias y los retos que las sociedades enfrentan para evitar la transmisión de infecciones como la Covid-19 (Capolongo et al., 2020; Sharifi y Khavarian-Garmsir, 2020).

Considerando la elevada concentración poblacional y las actividades económicas en las zonas urbanas, éstas se presentan como puntos críticos para la infección por Covid-19 (Sharifi y Khavarian-Garmsir, 2020: 1), pues se ha estimado que cerca de 90 por ciento de los casos de la enfermedad a nivel mundial se presentan en zonas urbanas (OMS, 2020). Un caso especial de los procesos de urbanización lo constituyen las zonas metropolitanas dado el elevado flujo económico-comercial y de personas que se acontece cada día.

La idea detrás de analizar la distribución, prevalencia e incidencia de la Covid-19 en las zonas urbanas o metropolitanas no es nueva. Diversas investigaciones han explorado las implicaciones socioeconómicas de la Covid-19 (Brodeur et al., 2020; Yang et al., 2020), los efectos de la infección sobre la movilidad en las ciudades (Abu-Rayash y Dincer, 2020; Iacus et al., 2020) o las oportunidades que en materia de planeación podrían desarrollarse (Capolongo et al., 2020; Sharifi y Khavarian-Garmsir, 2020), entre otros temas.

En el caso de México, la investigación se ha centrado en elementos como la epidemiología de la enfermedad (Suárez et al., 2020), la mortalidad relacionada (Bello-Chavolla et al., 2020; Parra-Bracamonte et al., 2020) o el análisis de las comorbilidades (Hernández-Garduño, 2020; Singer, 2020), por solo mencionar algunos temas. Además, un proyecto de análisis de la Covid-19 en México exploró la vulnerabilidad urbana de las zonas metropolitanas del país a la enfermedad de acuerdo con tres dimensiones: demográfica, de la salud y socioeconómica (Suárez Lastra et al., 2020). Este último trabajo encontró que 75.5 millones de habitantes residían en un municipio con vulnerabilidad media, 21 millones en vulnerabilidad alta y 22.8 millones en municipios con vulnerabilidad alta o crítica, 
lo cual reafirma la importancia de estudiar las características de las zonas metropolitanas ante escenarios como el expuesto por la Covid-19.

\section{Zonas Metropolitanas en México}

El fenómeno de la urbanización en México fue impulsado por el desarrollo económico lo que condujo a fenómenos como la concentración poblacional. Aunado a lo anterior, en poco más de un siglo, la población en México se multiplicó por 8.3 veces al pasar de 13.6 millones de personas en 1900 a 112.3 millones de individuos en 2010 (Sobrino, 2011). El proceso de urbanización en México se caracteriza por tres etapas (Garza, 2007: 78; Sobrino, 2011: 11):

La primera etapa abarca los años 1900 a 1940 y se caracterizó por un bajo crecimiento urbano, pues esta etapa se desarrolló en el contexto de la revolución mexicana y los enfrentamientos para conformar las bases del Estado actual.

La segunda etapa comprende los años 1940-1980 y se caracteriza por un acelerado crecimiento urbano impulsado por el cambio al modelo de Industrialización por Sustitución de Importaciones. Este cambio motivó cambios en el mercado laboral lo que generó modificaciones en el mercado laboral con un aumento de la secundarización y terciarización de las actividades, obligando a una masiva migración de zonas rurales a urbanas.

La tercera etapa se desarrolló a partir del año 1980 y se caracteriza por la aplicación del modelo económico neoliberal. En esta etapa se experimentan procesos de cambios como el definido por el crecimiento de las grandes urbes.

La tendencia a la metropolización en México ha sido impulsada por factores como la migración desde las áreas rurales a las ciudades, así como por la centralización del empleo, el desarrollo industrial y la producción manufacturera (Garza, 2007; Trejo Nieto, 2013). De manera tal que las zonas metropolitanas, áreas metropolitanas o metrópolis pueden entenderse como parte de un fenómeno que ha incentivado profundos cambios sociales, económicos y políticos, y por supuesto en la configuración de los territorios en los que existe "una extensión de la ciudad central más allá del municipio donde tuvo origen" (Trejo Nieto, 2013: 548).

Aunque existen diversas definiciones de lo que es una zona metropolitana, de acuerdo con la organismos como la Secretaría de Desarrollo Agrario Territorial y Urbano (SEDATU), el Consejo Nacional de Población (CONAPO) y el Instituto Nacional de Estadística y Geografía INEGI (INEGI) de México, ésta se define como 
el conjunto de dos o más municipios donde se localiza una ciudad de 100 mil o más habitantes, cuya área urbana, funciones y actividades rebasan los límites del municipio, incorporando dentro de su área de influencia directa a municipios vecinos, predominantemente urbanos, con los que mantiene un alto grado de integración socioeconómica. También se incluyen aquellos municipios que por sus características particulares son relevantes para la planeación y política urbana de las zonas metropolitanas en cuestión". Adicionalmente, se contempla... a los municipios con una ciudad de más de 500 mil habitantes; los que cuentan con ciudades de 200 mil o más habitantes ubicados en la franja fronteriza norte, sur y en la zona costera; y aquellos donde se asienten capitales estatales, estos últimos cuando no están incluidos en una zona metropolitana (SEDATU / CONAPO / INEGI, 2018).

En México el número de zonas metropolitanas se ha incrementado, pues en 2018 se definieron 74 zonas a partir de las 59 que se delimitaron en 2010. Esto plantea un fenómeno interesante ya que el aumento en el número de zonas metropolitanas en el país puede presentarse como efectos de la concentración poblacional en ciertas áreas geográficas del país y que se refleja en que las 74 zonas metropolitanas del país residen cerca de 62 por ciento de la población (SEDATU / CONAPO / INEGI, 2018). Es importante señalar que la metropolización puede entenderse como parte de los efectos propiciados por los procesos de industrialización y terciarización económica experimentados por el país con una fuerte presencia de la informalidad (Pradilla, 2016; González Arellano y Larralde Corona, 2019). Así, y si bien la relevancia de las zonas metropolitanas del país se define en términos de la articulación territorial y del funcionamiento económico, existen complejidades del sistema urbano que se presentan como reflejos de las diferencias económicas y sociales de los distintos espacios metropolitanos (Trejo Nieto, 2013).

Para este artículo se analizan las tres principales zonas metropolitanas de México: Zona Metropolitana de Monterrey, Zona Metropolitana de Guadalajara y Zona Metropolitana del Valle de México. La elección de estas zonas metropolitanas se debe a diversos aspectos asociados con la relevancia de su economía, así como con su concentración poblacional y los posibles efectos que sobre estas ha tenido la Covid-19 en términos de los casos confirmados y la mortalidad. Por un lado, estas tres zonas en el año 2015 concentraban casi 30 millones de personas que representaban cerca de 25 por ciento de la población total estimada en México. Por el otro, las zonas metropolitanas agrupan parte importante de los casos y defunciones 
por Covid-19, por lo que permite conocer un perfil general de la población afectada por la enfermedad.

De acuerdo con los elementos anteriores, este trabajo tiene como objetivo realizar un análisis comparativo de los casos confirmados, las defunciones y las prevalencias de enfermedades en infección por Covid-19 para las tres zonas metropolitanas en dos períodos. El primero es del 15 de julio al 15 de agosto y el segundo del 15 de noviembre al 15 de diciembre. La selección de estos dos periodos se debe a que son meses intermedios y permiten conocer y comparar en dos momentos diferenciados el comportamiento de la pandemia en las zonas metropolitanas de estudio.

Para realizar lo anterior el contenido de esta investigación se presenta en seis apartados adicionales al introductorio. En un segundo apartado se expone la metodología de la investigación. El tercer apartado contiene una breve descripción de las zonas metropolitanas estudiadas; mientras que en un cuarto apartado se presenta un análisis del contexto sociodemográfico de tales zonas. Los resultados del análisis de los casos confirmados y de la mortalidad se exponen en un quinto apartado, para en un apartado sexto presentar las conclusiones a las cuales se llegó.

\section{Metodología}

\section{Fuentes de datos}

Este estudio es de tipo descriptivo y utiliza datos de dos fuentes de información: la base de datos de casos asociados a la Covid-19 de la Dirección General de Epidemiología de la Secretaría de Salud de México (DGE / SSA, 2021) que en adelante se denomina base Covid-19, así como la Encuesta Intercensal de México 2015 (INEGI, 2015). La Encuesta Intercensal se empleó para dar un análisis del contexto sociodemográfico de las tres zonas metropolitanas en estudio. En cuanto a la base Covid-19, ésta se usó para conocer la distribución de casos confirmados de la enfermedad, las muertes registradas y las prevalencias de diabetes, hipertensión, obesidad o enfermedades cardiovasculares entre personas con Covid-19 en dos periodos de análisis: 15 de junio al 15 de agosto de 2020 y del 15 de noviembre al 15 de diciembre del mismo año. En el caso de la base Covid-19 debe considerarse que esta información se actualiza diariamente.

\section{Definición de variables}

Los casos confirmados de Covid-19 se identificaron acorde con los resultados del análisis por asociación clínica epidemiológica, por comité de dic- 
taminación o por la aplicación de muestra de laboratorio o prueba antígena y resultado positivo a SARS-CoV-2 (DGE / SSA, 2021). Los cortes temporales del análisis se hicieron de acuerdo con la fecha de actualización de los datos. Las enfermedades que se estudiaron fueron: diabetes, hipertensión, obesidad o enfermedades cardiovasculares según diagnóstico del paciente y disponibles en la misma base Covid-19. Estas enfermedades se definieron porque además de identificarse en la literatura relacionada (Ejaz et al., 2020; Gold et al., 2020) entre la población mexicana constituyen las principales causas de morbilidad o de mortalidad.

Otras variables que se emplearon en esta investigación son de tipo sociodemográfico: sexo (mujer, hombre) y grupo etario (0-14 años, 15-59 años, 60 y más años) de la persona registrada en la base Covid-19 dado que los análisis de los casos confirmados y la defunciones por la infección se analizan estratificando por estas variables. En cuanto a la caracterización sociodemográfica de las zonas metropolitanas estudiadas se agregan la escolaridad según niveles (sin escolaridad, básico, medio superior, superior, posgrado) y la situación conyugal (casado(a) / unido(a), divorciado(a) / separado(a), viudo(a), soltero(a)).

\section{Análisis de datos}

El análisis de los datos consiste en la estimación de prevalencias de casos confirmados de la enfermedad de acuerdo con la expresión.

$$
\mathrm{P}=(\mathrm{A} / \mathrm{B}) * 100
$$

Donde:

$\mathrm{P}_{\mathrm{t}}=$ Prevalencia

$\mathrm{t}=1,2$ (periodo 1 ( 15 de julio al 15 de agosto), periodo 2 ( 15 de noviembre al 15 de diciembre).

$\mathrm{A}_{\mathrm{n}}=$ Número de defunciones por Covid-19 con la enfermedad $n$.

$\mathrm{n}=1,2,3,4$ (diabetes, hipertensión, obesidad y cardiovascular).

$\mathrm{B}=$ Número de defunciones totales por Covid-19.

Tanto el análisis de los casos confirmados como de la mortalidad se realizó estratificando por sexo, por grupo etario y para las trez zonas metropolitanas del estudio. Definida la metodología de la investigación, a continuación se presenta una caracterización de las tres zonas metropolitanas en análisis. 
Análisis comparativo de los casos confirmados y defunciones por Covid-19 en la población ... / D. LOZANO KEYMOLEN et al.

\section{RESUltados}

\section{Análisis sociodemográfico de las zonas metropolitanas}

a) Zona Metropolitana de Monterrey (ZMMTY)

La Zona Metropolitana de Monterrey (ZMMTY) (Figura 1) se localiza al norte de México y está conformada por 18 municipios del Estado de Nuevo León. En el año 2015 esta zona metropolitana contaba con 4.6 millones de habitantes, y se caracterizaba por ser uno de los centros industriales de México. Sin embargo, en los últimos años la manufactura y el sector servicios han ido ganando terreno, por lo que actualmente esta zona es la segunda con mayor aportación al Producto Interno Bruto (PIB) nacional, lo que indica la importancia que tiene esta zona en todo el país.

\section{b) Zona Metropolitana de Guadalajara}

La Zona Metropolitana de Guadalajara (ZMGDL) (Figura 2) es una de las tres zonas definidas para el Estado de Jalisco, México, en conjunto con la zona metropolitana de Ocotlán y la de Puerto Vallarta, si bien ésta última comparte extensión con el Estado de Nayarit. Específicamente, la ZMGDL está compuesta por 10 municipios de Jalisco integrados por factores como la conurbación física, la integración funcional o la política urbana. Con una extensión de cerca de 3,600 $\mathrm{km}^{2}$, para el año 2015, residían 4,887,383 personas en la ZMGDL (SEDATU / CONAPO / INEGI, 2018). Sin embargo, esta zona metropolitana ha experimentado un proceso de disminución en su crecimiento poblacional ya que la tasa de crecimiento medio anual entre 1990 y 2000 fue de 2.1 por ciento, mientras que en el periodo 20002010 fue de 1.8 y de 1.6 por ciento entre los años 2010 y 2015 (SEDATU / CONAPO / INEGI, 2018).

\section{c) Zona Metropolitana del Valle de México}

La Zona Metropolitana del Valle de México (ZMVM) (Figura 3) tiene su base territorial y poblacional en la Ciudad de México, sin embargo, el crecimiento de las zonas urbanas y de esta ciudad a partir de los años cincuenta provocaron el surgimiento de zonas metropolitanas, la primera de ellas fue la ZMVM. A partir de la década de los noventa del siglo XX, los principales crecimientos poblacionales y espaciales alrededor de la Ciudad de México generaron la extensión de la ZMVM hacia el Estado de México. Según Pradilla (2016) estos cambios se dieron en consonancia con la implementación del modelo económico neoliberal. 

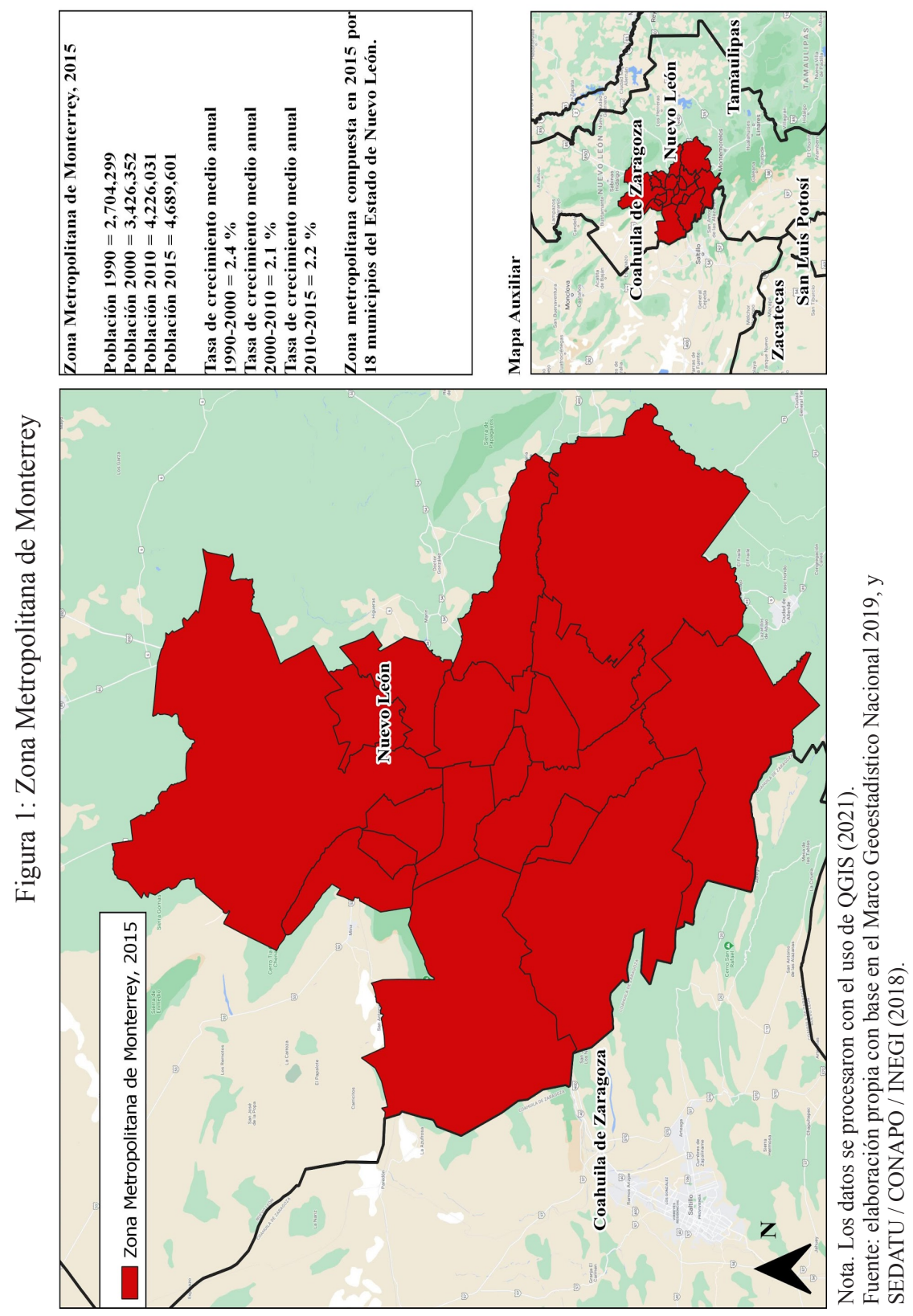
Análisis comparativo de los casos confirmados y defunciones por Covid-19 en la población ... / D. LOZANO KEYMOLEN et al.

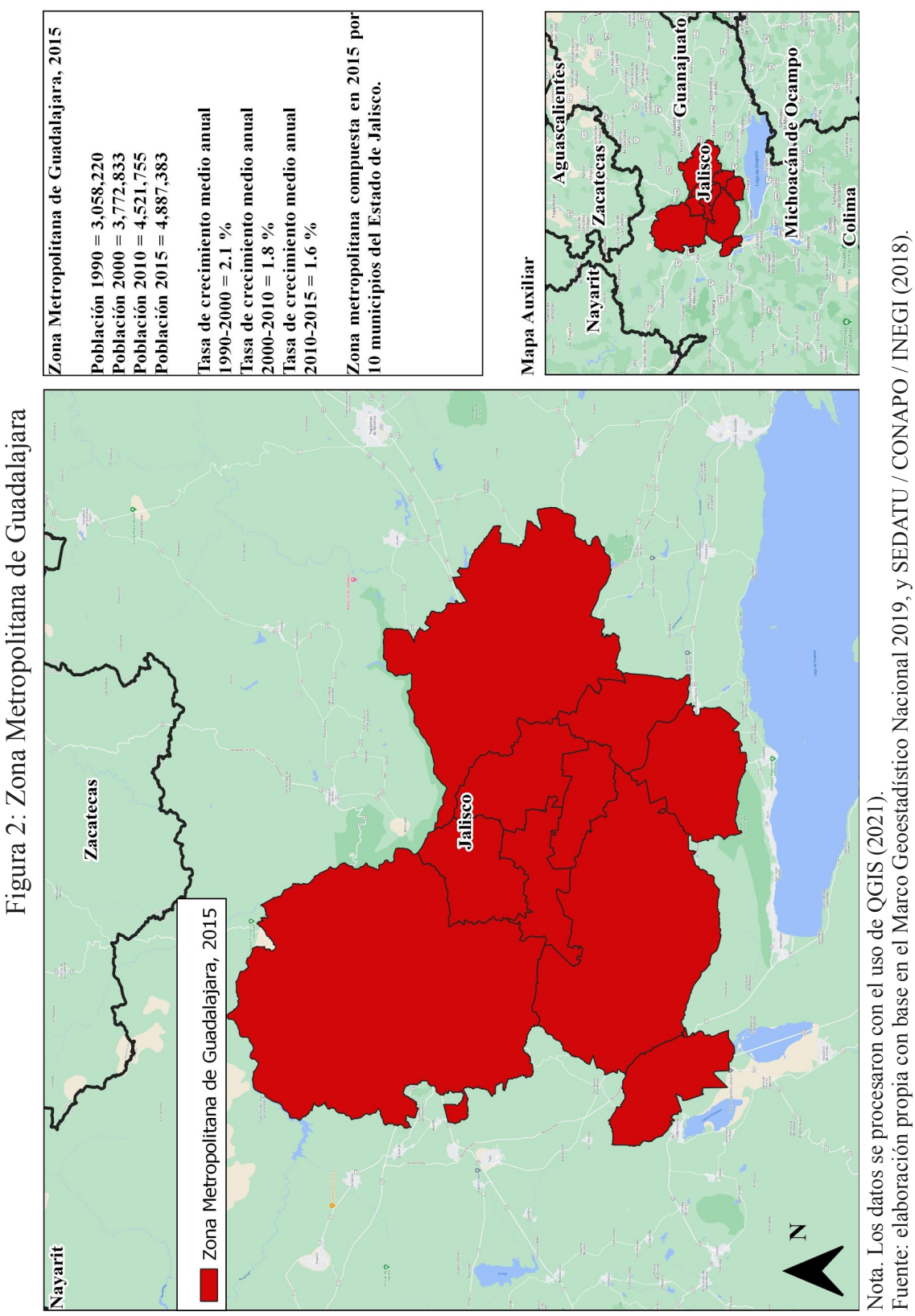




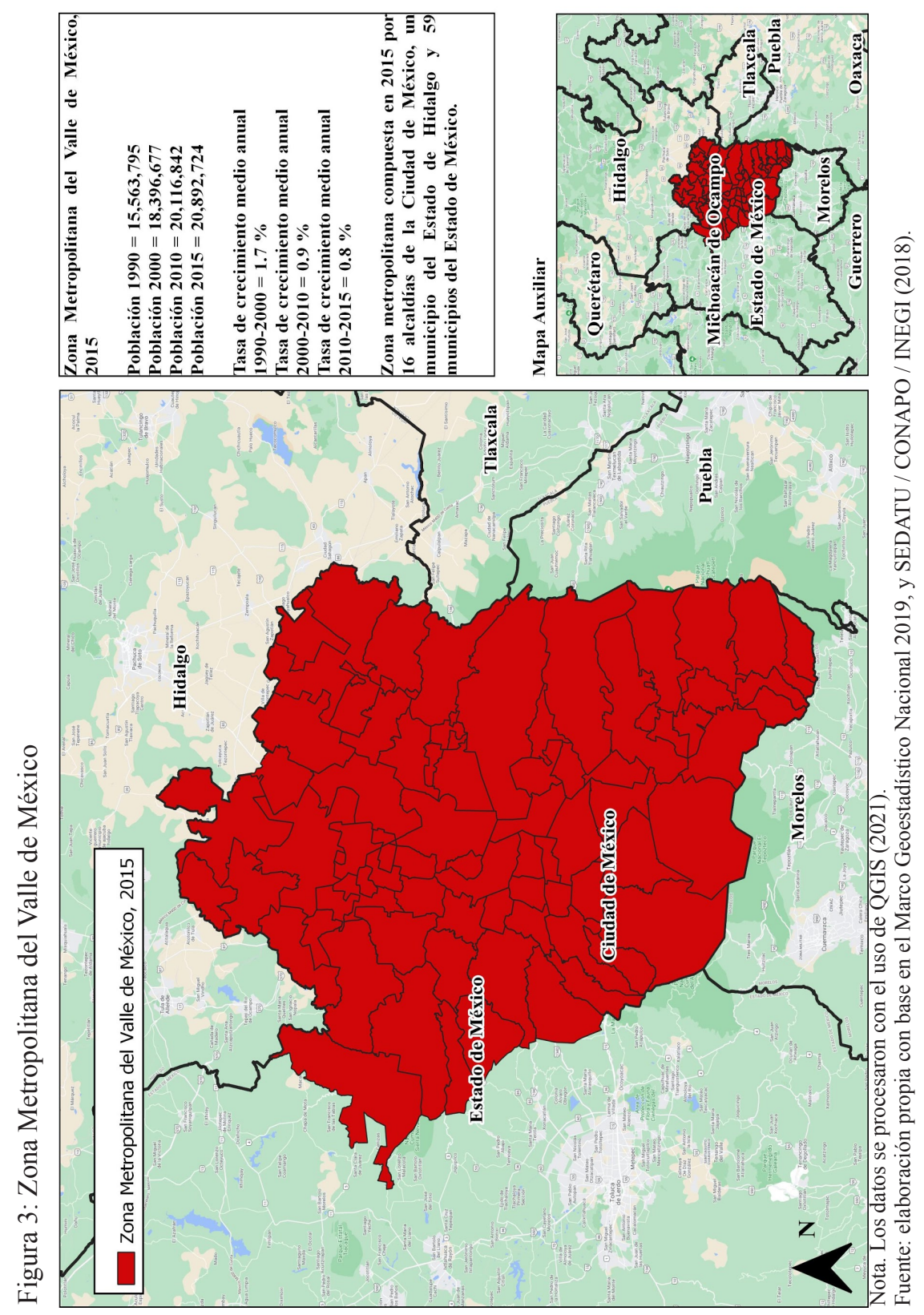


La ZMVM se ha expandido a través del tiempo y hasta el año 2005 se plantea una definición oficial en la que esta zona está conformada por 16 alcaldías de la ciudad de México, 59 municipios del Estado de México y un municipio del Estado de Hidalgo (SEDATU / CONAPO / INEGI, 2018) lo que no representa un cambio para las definiciones de 2010 y de 2015. En el 2015, la ZMVM tiene aproximadamente 20.9 millones de habitantes, lo que la sitúa entre las diez ciudades más grandes del mundo y la primera de México. Respecto de su economía, la principal actividad de la ZMVM es el sector terciario, en particular, esta zona tiene una predominancia del sector comercio y los servicios al productor. Es importante señalar que la ZMVM es la mayor aportadora al PIB a nivel nacional, lo que ratifica la importancia de esta urbe en lo que respecta a los aspectos poblacionales y económicos.

\section{Contexto sociodemográfico de las zonas metropolitanas}

Como se comentó, las tres zonas metropolitanas que se estudian se caracterizan por ser las más pobladas del país, por lo que resulta interesante conocer la estructura poblacional de estas urbes. En particular se analizan las variables socidoemográficas como el sexo, la edad (grupos etarios), la escolaridad y la situación conyugal a las poblaciones estimadas en 2015 con datos de la Encuesta Intercensal (ver apartado de Metodología).

En este contexto, la Tabla 2 expone los resultados del análisis sociodemográfico. En cuanto al sexo, la ZMVM expone un mayor componente de mujeres que de hombres en comparación con las otras dos zonas estudiadas. Respecto de la edad, tanto la ZMGDL como la ZMMTY presentan un mayor componente de población de 0 a 14 años que la ZMVM, y en contraste, esta última zona tuvo una mayor componente de población entre las edades de 15-59 años. En cuanto a las variables de la escolaridad y la situación conyugal, los resultados muestran que la ZMGDL tenía el mayor porcentaje de población sin escolaridad (10.1 por ciento). En cuanto a la situación conyugal, se observó que la ZMMTY tenía el mayor porcentaje de personas casadas o unidas que la ZMVM o la ZMGDL.

Lo anterior permite mostrar la configuración poblacional en las zonas metropolitanas que presentan diferencias en las variables sociodemográficas que podrían impactar en el número de casos confirmados o en la mortalidad por Covid-19 de estas poblaciones, por ejemplo, el mayor porcentaje de adultos mayores en la ZMVM impacta en una alta mortalidad de este grupo etario por la enfermedad. 
Tabla 2: Distribución porcentual de la población total según variables sociodemográficas en la ZMMTY, la ZMGDL y la ZMVM, 2015

\begin{tabular}{|c|c|c|c|c|c|c|}
\hline \multirow{2}{*}{$\begin{array}{l}\text { Variables } \\
\text { sociodemográficas } \\
\text { Sexo }\end{array}$} & \multicolumn{2}{|c|}{$\begin{array}{c}\text { Zona Metropolitana } \\
\text { de Monterrey }\end{array}$} & \multicolumn{2}{|c|}{$\begin{array}{c}\text { Zona Metropolitana } \\
\text { de Guadalajara }\end{array}$} & \multicolumn{2}{|c|}{$\begin{array}{l}\text { Zona Metropolitana } \\
\text { del Valle de México }\end{array}$} \\
\hline & Población & $\%$ & Población & $\%$ & Población & $\%$ \\
\hline Mujer & $2,362,995$ & 50.4 & $2 ’ 501,352$ & 51.2 & $10^{\prime} 027,191$ & 48.0 \\
\hline Hombre & $2,326,606$ & 49.6 & $2 ’ 386,031$ & 48.8 & $10^{\prime} 865,533$ & 52.0 \\
\hline Total $(100 \%)$ & $4,689,601$ & 100.0 & 4'887,383 & 100.0 & $20^{\prime} 892,724$ & 100.0 \\
\hline \multicolumn{7}{|l|}{ Grupo de edad } \\
\hline 0 a 14 & 1’211,835 & 25.8 & $1 ’ 322,058$ & 27.1 & $4^{\prime} 804,425$ & 23.0 \\
\hline 15 a 59 & 3’030,339 & 64.6 & 3'094,140 & 63.3 & $13^{\prime} 637,846$ & 65.3 \\
\hline 60 y más & 442,724 & 9.4 & 469,099 & 9.6 & $2 ’ 427,372$ & 11.6 \\
\hline No especificado & 4,703 & 0.1 & 2,086 & 0.0 & 23,081 & 0.1 \\
\hline Total $(100 \%)$ & 4’689,601 & 100.0 & $4^{\prime} 887,383$ & 100.0 & $20 ’ 892,724$ & 100.0 \\
\hline \multicolumn{7}{|l|}{ Escolaridad } \\
\hline Sin escolaridad & 409,965 & 9.2 & 480,851 & 10.4 & $1^{\prime} 707,369$ & 8.5 \\
\hline Básico & 2’286,661 & 51.3 & $2^{\prime} 474,615$ & 53.4 & $9^{\prime} 815,444$ & 49.0 \\
\hline Media superior & 954,488 & 21.4 & 896,243 & 19.3 & $4^{\prime} 803,130$ & 24.0 \\
\hline Superior & 683,750 & 15.3 & 692,976 & 14.9 & 3’'185,444 & 15.9 \\
\hline Posgrado & 94,297 & 2.1 & 76,756 & 1.7 & 413,812 & 2.1 \\
\hline No especificado & 28,031 & 0.6 & 15,884 & 0.3 & 113,658 & 0.6 \\
\hline Total $(100 \%)$ & 4’457,192 & 100.0 & $4^{\prime} 637,325$ & 100.0 & $20^{\prime} 038,857$ & 100.0 \\
\hline \multicolumn{7}{|l|}{ Situación conyugal } \\
\hline $\begin{array}{l}\text { Casado(a) o unión } \\
\text { libre }\end{array}$ & $2,104,107$ & 56.4 & $1 ’ 986,015$ & 51.7 & $8^{\prime} 866,290$ & 51.8 \\
\hline $\begin{array}{l}\text { Divorciada(o) o } \\
\text { separada(o) }\end{array}$ & 216,562 & 5.8 & 231,331 & 6.0 & $1 ’ 276,043$ & 7.5 \\
\hline Viuda(o) & 146,820 & 3.9 & 164,514 & 4.3 & 835,624 & 4.9 \\
\hline Soltera(o) & $1 ’ 248,241$ & 33.5 & $1 ’ 447,269$ & 37.7 & 6’037,982 & 35.3 \\
\hline No especificado & 12,873 & 0.3 & 9,856 & 0.3 & 88,244 & 0.5 \\
\hline Total $(100 \%)$ & 3’728,603 & 100.0 & 3'838,985 & 100.0 & $17^{\prime} 104,183$ & 100.0 \\
\hline
\end{tabular}

Fuente: elaboración propia con base en la Encuesta Intercensal 2015.

\section{Análisis de los casos confirmados de Covid-19}

La Tabla 3 resume el análisis exploratorio de los casos confirmados de Covid-19 en las tres zonas metropolitanas y para los dos periodos en análisis. En cuanto al sexo, destaca tanto para la ZMMTY y la ZMGDL un aumento entre periodos en el procentaje de mujeres con casos confirmados de la 
enfermedad. Por ejemplo, en la ZMMTY el cambio fue de 48.7 a 51.6 por ciento, mientras que en la ZMGDL hubo un aumento de 50.6 a 52.1 por ciento entre periodos. A diferencia de las dos zonas anteriores, el porcentaje de casos confirmados para la ZMVM es similar.

Cuando se analizan los casos confirmados por grupo etario, es posible determinar que la ZMMTY tuvo un aumento para las edades 15-59 al pasar de 79.1 a 83.1 por ciento, pero la ZMGDL y la ZMVM no mostraron cambios aparentes. En cuanto al grupo etario que concentra a las personas de edades avanzadas, el comportamiento entre zonas metropolitanas es heterogéneo. Así, la ZMGDL no indicó cambios en el porcentaje de casos confirmados por Covid-19, en cambio, la ZMMTY mostró una disminución del pasar de 19.7 a 15.4 por ciento. Finalmente, la ZMVM tuvo un incremento en el porcentaje de casos confirmados entre personas con 60 o más años de edad (de 17.7 a 19.3 por ciento). Es importante señalar que el alto porcentaje de infectados en las edades de 15 a 59 años en todas las zonas metropolitanas se debe a la alta movilidad de esta población por encontrarse en las edades productivas, sin embargo, según la OPS (2020) este grupo poblacional tiene menor riesgo de mortalidad.

Tabla 3: Distribución porcentual de la población confirmada con Covid-19 según variables sociodemográficas, en la ZMMTY, la ZMGDL y la ZMVM, 2020

Variables Zona Metropolitana de Monterrey

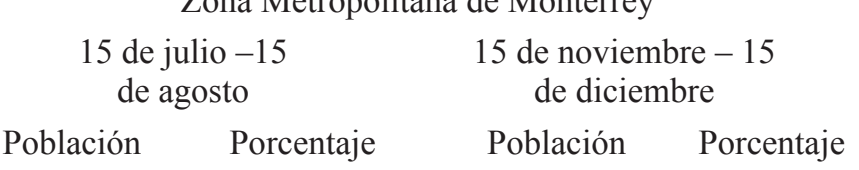

Sexo

Mujer

4,759

48.7

5,360

51.6

Hombre

5,022

51.3

5,020

Total

9,781

100.0

10,380

100.0

Grupos de edad

0-14 años

114

1.2

150

15-59 años

7,741

79.1

8,627

83.1

60 o más años

1,926

19.7

1,603

15.4

Total

9,781

15.4

10,380

100.0 
Tabla 3: Continuación

Variables

$$
\text { Zona Metropolitana de Guadalajara }
$$

$$
\begin{array}{ccc}
\multicolumn{2}{c}{\begin{array}{cc}
15 \text { de julio }-15 \\
\text { de agosto }
\end{array}} & \begin{array}{c}
15 \text { de noviembre }-15 \\
\text { de diciembre }
\end{array} \\
\text { Población } & \text { Porcentaje } & \text { Población Porcentaje }
\end{array}
$$

Sexo

Mujer

2,073

50.6

2,902

52.1

Hombre

2,025

49.4

2,671

Total

4,098

100.0

5,573

100.0

Grupos de edad

0-14 años

44

1.1

60.0

15-59 años

3,147

76.8

4,295.0

60 o más años

907

22.1

$1,218.0$

Total

4,098

21.9

5,573

100.0

Tabla 3: Continuación

Variables

Zona Metropolitana del Valle de México

$$
\begin{array}{cc}
15 \text { de julio }-15 & 15 \text { de noviembre }-15 \\
\text { de agosto } & \text { de diciembre }
\end{array}
$$

Población Porcentaje Población Porcentaje

Sexo

Mujer

18,238

50.6

18,622

50.9

Hombre

17,820

49.4

17,962

Total

36,058

100.0

36,584

100.0

Grupos de edad

0-14 años

$$
1,214
$$

3.4

886

15-59 años

28,476

79.0

28,649

78.3

60 o más años

6,368

17.7

7,049

19.3

Total

36,058

19.3

36,584

100.0

Notas. ZMMTY = Zona Metropolitana de Monterrey, ZMGDL = Zona Metropolitana de Guadalajara, ZMVM = Zona Metropolitana del Valle de México.

Fuente: elaboración propia con base en datos de la Dirección General de Epidemiología de la Secretaría de Salud de México. 
Una vez se ha descrito la distribución de la Covid-19 entre los periodos de análisis y para las zonas metropolitanas estudiadas, en el siguiente apartado se resumen los resultados del análisis de las defunciones.

\section{Defunciones por Covid-19}

En la Tabla 4 se resumen los resultados del análisis realizado a las defunciones por la enfermedad en las zonas metropolitanas estudiadas. Respecto del sexo, un mayor porcentaje de las muertes entre hombres se identificaron para la ZMVM, pero existe un descenso en este procentaje entre los periodos estudiados (de 66 a 61.5 por ciento). Estos resultados no indicaron cambios entre periodos para la ZMMTY y la ZMGDL en cuanto a la distribución por sexo de las defunciones por Covid-19.

Tabla 4: Distribución porcentual de las defunciones por Covid-19 según variables sociodemográficas, en la ZMVM, ZMG y ZMM, 2020

Variables

Sexo

Mujer

Hombre

Total

Grupos de edad

0-14 años

15-59 años

60 o más años

Total
Zona Metropolitana de Monterrey

$$
15 \text { de julio }-15
$$

de agosto

$$
\text { Población Porcentaje }
$$

15 de noviembre -15 de diciembre Población Porcentaje 
Tabla 4: Continuación

Variables

Zona Metropolitana de Guadalajara

$$
\begin{gathered}
15 \text { de julio - } 15 \\
\text { de agosto }
\end{gathered}
$$

Porcentaje

15 de noviembre - 15

de diciembre

Sexo

$\begin{array}{lrrrr}\text { Mujer } & 205 & 37.5 & 109 & 37.7 \\ \text { Hombre } & 341 & 62.5 & 180 & 62.3 \\ \text { Total } & 546 & 100.0 & 289 & 100.0\end{array}$

Grupos de edad

0-14 años

3

0.5

0.0

0.0

15-59 años

175

32.1

85.0

29.4

60 o más años

368

67.4

204.0

70.6

Total

546

21.9

289

100.0

Tabla 4: Continuación

Variables

Zona Metropolitana del Valle de México

15 de julio - 15
de agosto
15 de noviembre - 15

de diciembre

Población Porcentaje Población Porcentaje

Sexo

Mujer 865

34.0

881

38.5

Hombre

1,676

66.0

1,407

61.5

Total

2,541

100.0

2,288

100.0

Grupos de edad

0-14 años

8

0.3

3

0.1

15-59 años

871

34.3

770

60 o más años

1,662

65.4

1,515

66.2

Total

2,541

19.3

2,288

100.0

Notas. ZMMTY = Zona Metropolitana de Monterrey, ZMGDL = Zona Metropolitana de Guadalajara, ZMVM = Zona Metropolitana del Valle de México.

Fuente: elaboración propia con base en datos de la Dirección General de Epidemiología de la Secretaría de Salud de México.

Un comportamiento similar se observó para la ZMGDL en la que entre personas de 15-59 años disminuyó el porcentaje de muertes por Covid-19 
al pasar de 32.1 a 29.4 por ciento, mientras que entre las edades de 60 o más años hubo un aumento de 67.4 a 70.6 por ciento. A diferencia de la ZMMTY y la ZMGDL, para la ZMVM no se estimaron cambios notables en el porcentaje de defunciones por Covid-19 entre grupos de edad.

Siguiendo con el análisis de las defunciones registradas por Covid-19, en el siguiente apartado se resumen los resultados del análisis de las muertes de acuerdo con la presencia de diabetes, hipertensión, obesidad, enfermedades cardiovasculares.

\section{Enfermedades entre defunciones por Covid-19}

En la Tabla 5 se presentan los resultados del análisis de las muertes por Covid-19 de acuerdo con la presencia de diabetes, hipertensión, obesidad o enfermedades cardiovasculares. El comportamiento de las defunciones entre periodos indica diferencias entre las zonas metropolitanas, destacando los casos de la ZMMTY y de la ZMGDL que tuvieron resultados contrastantes. Es notorio que la ZMMTY presentó descensos en las defunciones por Covid-19 y las enfermedades estudiadas, pues, por ejemplo, las muertes por Covid-19 y con diabetes en la persona descendieron de 38.7 a 27.7 por ciento.

Tabla 5: Distribución porcentual de defunciones por Covid-19 y comorbilidades específicas en la ZMMTY, la ZMGDL y la ZMVM, 2020

Variables

Zona Metropolitana de Monterrey

\begin{tabular}{lrrrr} 
& \multicolumn{2}{c}{$\begin{array}{c}15 \text { de julio }-15 \\
\text { de agosto }\end{array}$} & \multicolumn{2}{c}{15 de noviembre -15} \\
& Pe diciembre \\
& Población & Porcentaje & Población & Porcentaje \\
Neumonía & 972 & 89.3 & 163 & 40.3 \\
Diabetes & 421 & 38.7 & 112 & 27.7 \\
Hipertensión & 508 & 46.6 & 129 & 31.9 \\
Obesidad & 253 & 23.2 & 52 & 12.9 \\
$\begin{array}{l}\text { Enfermedades } \\
\text { cardiovasculares }\end{array}$ & 52 & 4.8 & 15 & 3.7 \\
Total defunciones & 1,089 & & & \\
Covid-19 & & & 404 &
\end{tabular}

En cuanto a la ZMGDL, los resultados indican aumentos en todas las enfermedades analizadas entre las personas fallecidas por Covid-19, pues, por ejemplo, la diabetes se incrementó de 47.1 a 56.7 por ciento o el caso 
de la obesidad con un aumento de 28 al 34.9 por ciento. Por último, en la ZMVM solo la diabetes (de 36.2 a 39.7 por ciento) mostró cambios entre los periodos estudiados.

Tabla 5: Continuación

Variables

Zona Metropolitana de Guadalajara

\begin{tabular}{lrrrr} 
& \multicolumn{2}{c}{$\begin{array}{c}15 \text { de julio }-15 \\
\text { de agosto }\end{array}$} & \multicolumn{2}{c}{ 15 de noviembre -15} \\
& Población diciembre \\
& 352 & Porcentaje & Población & Porcentaje \\
Neumonía & 257 & 47.5 & 202 & 69.9 \\
Diabetes & 298 & 54.6 & 164 & 56.7 \\
Hipertensión & 153 & 28.0 & 101.0 & 79.9 \\
Obesidad & 27 & 4.9 & 28 & 9.9 \\
$\begin{array}{l}\text { Enfermedades } \\
\text { cardiovasculares }\end{array}$ & & & & \\
$\begin{array}{l}\text { Total defunciones } \\
\text { Covid-19 }\end{array}$ & 546 & & 289 &
\end{tabular}

Tabla 5: Continuación

Variables

Zona Metropolitana del Valle de México

\begin{tabular}{lrrrr} 
& \multicolumn{2}{c}{$\begin{array}{c}15 \text { de julio }-15 \\
\text { de agosto }\end{array}$} & \multicolumn{2}{c}{15 de noviembre -15} \\
& de diciembre \\
& Población & Porcentaje & Población & Porcentaje \\
Neumonía & 2,004 & 78.9 & 1,520 & 66.4 \\
Diabetes & 920 & 36.2 & 795 & 34.7 \\
Hipertensión & 1,040 & 40.9 & 942 & 41.2 \\
Obesidad & 507 & 20.0 & 448 & 19.6 \\
$\begin{array}{l}\text { Enfermedades } \\
\text { cardiovasculares }\end{array}$ & 115 & 4.5 & 103 & 4.5 \\
Total defunciones & 2,541 & & & \\
Covid-19 & & & 2,288 &
\end{tabular}

Notas. ZMMTY = Zona Metropolitana de Monterrey, ZMGDL = Zona Metropolitana de Guadalajara, ZMVM = Zona Metropolitana del Valle de México.

Fuente: elaboración propia con base en datos de la Dirección General de Epidemiología de la Secretaría de Salud de México. 
Análisis comparativo de los casos confirmados y defunciones por Covid-19 en la población ... / D. LOZANO KEYMOLEN et al.

\section{DiSCUSIÓN Y CONCLUSIONES}

A lo largo de la historia, fenómenos como la guerra, la hambruna y las enfermedades han moldeado gran parte de los hechos sociales, económicos, políticos y culturales de la humanidad. Estos fenómenos han sido derivados de diversas interacciones de las personas o las comunidades entre sí, y en el caso específico de las enfermedades, existen importantes ejemplos de interacciones humanas con el medio. Incluso, la actual pandemia causada por la Covid-19 ha sido señalada como consecuencia de la interacción del hombre con el medio ambiente, específicamente con la fauna silvestre.

Desde que se confirmó el primer caso de Covid-19, la humanidad ha experimentado diversas consecuencias entre las que destacan la elevada morbilidad y mortalidad por la enfermedad, la insuficiencia de los recursos materiales y médicos para enfrentar la pandemia o la disminución o detención de las actividades sociales y económicas. Las consecuencias de lo anterior son amplias y algunas todavía no se manifiestan en toda su intensidad. Sin embargo, entre las más sobresalientes de estas consecuencias, se encuentran los efectos de la concentración humana como resultado de la vulnerabilidad de la población residente en las zonas urbanas a la Covid-19.

México ha experimentado un proceso de urbanización asociado con cambios en el modelo económico, la industrialización y la transformación de las actividades productivas o la migración del campo a las ciudades, entre otros factores (González Arellano y Larralde Corona, 2019). Este proceso de urbanización puede resumirse en tres etapas (Garza, 2007; Sobrino, 2011), sin embargo, aquella etapa iniciada con el cambio al modelo económico neoliberal muestra las diferencias o contrastes socioeconómicos con mayor intensidad, así como de la carencia o ausencia de condiciones de habitabilidad de estas zonas. Considerando el acelerado proceso de urbanización en México, este proceso se ha caracterizado además por mostrar una tendencia a la metropolización (Garza, 2007; Trejo Nieto, 2013).

Entonces, las zonas metropolitanas se han identificado como áreas en las que el contagio de enfermedades como la provocada por el coronavirus SARS-CoV-2 se favorece, entre otros factores, por la elevada concentración poblacional, el flujo de personas y actividades, las carencias de servicios, la presencia de servicios deficientes o ausentes de agua potable, saneamiento y limpieza (Reyes et al., 2013; Capolongo et al., 2020; Lee et al., 2020; Sharifi y Khavarian-Garmsir, 2020). 
A partir de elementos como lo anteriores, este trabajo analizó la distribución de los casos confirmados y las defunciones por Covid-19 en la zona metropolitana de Monterrey, la zona metropolitana de Guadalajara y la zona metropolitana del Valle de México. En este caso las zonas metropolitanas más pobladas de México según datos de la Encuesta Intercensal 2015. Mientras que la misma Encuesta Intercensal indicó diferencias en la composición por sexo y grupo etario entre las zonas de estudio, el análisis de los datos sobre la Covid-19 indican aspectos trascendentales.

Primero, en las zonas metropolitanas estudiadas, los casos confirmados de la Covid-19 por sexo exponen un aumento para las mujeres en la ZMMTY y lo mismo para la ZMGDL, pero no en la ZMVM. Este hecho expone que los casos confirmados por Covid-19 son superiores en la ZMGDL y la ZMMTY en las mujeres que en los hombres. De acuerdo con la edad, los datos indican un mayor diagnóstico de Covid-19 entre las personas de edades 15-59 años en las zonas analizadas. Sobre esto, se puede hipotetizar sobre posibles efectos de la movilidad sobre el contagio de la enfermedad, pues a pesar de la disminución de las actividades educativas y laborales, este grupo poblacional es el denominado en edades productivas.

Segundo, las muertes por Covid-19 en las zonas metropolitanas presentan un mayor componente de hombres, aunque en la ZMVM se estimó un aumento de las defunciones entre mujeres. Esto indicaría que las diferencias entre sexos pueden disminuir. En cuanto a las defunciones por edad, las personas adultas mayores no solo tienen el mayor componente de muertes, sino que también existe un aumento entre periodos para las tres zonas metropolitanas, lo cual puede ser indicativo del efecto de la edad avanzada en la mortalidad por Covid-19 (Mueller et al., 2020).

Tercero, el análisis de las prevalencias de diabetes, hipertensión, obesidad o enfermedades cardiovasculares entre las muertes por Covid-19 expone una situación compleja para las tres zonas metropolitanas. Mientras la ZMGDL y la ZMMTY tuvieron aumentos en las prevalencias de las enfermedades entre las defunciones por Covid-19, la ZMVM solo mostró un incremento en la prevalencia de diabetes. Aunque se ha comentado sobre esto, debe considerarse que la población mexicana experimenta elevadas prevalencias de enfermedades como diabetes, hipertensión u obesidad, los cuales han sido identificados como potenciadores de la infección por Covid-19. En México, la diabetes se estima afecta a 9.5 por ciento de los adultos con 20 o más años de edad, aunque un adicional 4.1 por ciento no estaría diagnosticado lo que llevaría a una prevalencia de 13.7 por ciento (Basto-Abreu et al., 2020). También, se estima que entre personas de 20 
y más años de edad, 36.1 por ciento tendría obesidad de acuerdo con el índice de masa corporal (Barquera et al., 2020), así como que 25.5 por ciento tendría hipertensión arterial (Campos-Nonato, Hernández-Barrera, Pedroza-Tobías, Medina y Barquera, 2018).

Esta investigación no tiene elementos para determinar los factores que incentivan los cambios en el porcentaje de casos confirmados o de las defunciones por Covid-19 en algunas áreas como la ZMMTY. Sin embargo, se puede hipotetizar que esto se relacionaría con factores como la disponibilidad de infraestructura de atención y personal médico para la enfermedad, así como por determinados comportamientos sociales que incentivan el contacto entre personas.

Este trabajo presenta diversas limitaciones asociadas con los datos empleados y los procedimientos de análisis realizados. En cuanto a este último punto, el análisis podría ampliarse en futuros estudios a todas las zonas urbanas del país o en comparativas con las áreas semiurbanas y rurales, así como lograr una comparativa temporal sobre las tendencias de los casos confirmados y las defunciones.

En conclusión, esta investigación muestra que la epidemia por Covid-19 en México es diferenciada entre las principales zonas metropolitanas del país. Si bien se ha comentado sobre el efecto positivo que el plan de vacunación tendría, la comparativa temporal realizada indica que la aparente brecha entre sexos para los casos confirmados se ha acotado. Adicionalmente, se destaca que a pesar de diferencias en la proporción de muertes, las personas de edades avanzadas representan el grupo poblacional con el mayor porcentaje de las muertes registradas. A pesar de lo anterior, sin lugar a dudas la investigación sobre la Covid-19 aun tiene múltiples interrogantes que resolver y que son conocimiento para enfrentar las futuras epidemias-pandemias que países como México experimentará.

\section{REFERENCIAS BIBLIOGRÁFICAS}

Abu-Rayash, A., y Dincer, I., 2020, “Analysis of mobility trends during the COVID-19 coronavirus pandemic: Exploring the impacts on global aviation and travel in selected cities", en Energy Research \& Social Science, 68, 101693, disponible en https://doi.org/10.1016/j.erss.2020.101693

Barquera, S., Hernández-Barrera, L., Trejo-Valdivia, B., Shamah, T., Campos-Nonato, I., y Rivera-Dommarco, J., 2020, “Obesidad en México, prevalencia y tendencias en adultos", en Salud Pública de México, 62(6), 682-692, disponible en https://doi.org/10.21149/11630

Basto-Abreu, A., Barrientos-Gutiérrez, T., Rojas-Martínez, R., Aguilar-Salinas, C. A., López-Olmedo, N., De la Cruz-Góngora, V., Rivera-Dommarco, J., Sha- 
mah-Levy, T., Romero-Martínez, M., Barquera, S., López-Ridaura, R., Hernández-Ávila, M., y Villalpando, S., 2020, Prevalencia de diabetes y descontrol glucémico en México: resultados de la Ensanut 2016. Salud Publica de México, 62(1), 50-59, disponible en https://doi.org/10.21149/10752

Bello-Chavolla, O. Y., Bahena-López, J. P., Antonio-Villa, N. E., Vargas-Vázquez, A., González-Díaz, A., Márquez-Salinas, A.... Aguilar-Salinas, C. A., 2020, "Predicting Mortality Due to SARS-CoV-2: A Mechanistic Score Relating Obesity and Diabetes to Covid-19 Outcomes in Mexico", en The Journal of Clinical Endocrinology and Metabolism, 105(8), dgaa346, disponible en https://doi.org/10.1210/ clinem/dgaa346

Boyce, M. R., Katz, R., y Standley, C. J., 2019, “Risk Factors for Infectious Diseases in Urban Environments of Sub-Saharan Africa: A Systematic Review and Critical Appraisal of Evidence", en Tropical Medicine and Infectious Disease, 4(4), 123, disponible en https://doi.org/10.3390/tropicalmed4040123

Brodeur, A., Gray, D., Islam, A., y Bhuiyan, S. J., 2020, A Literature Review of the Economics of Covid-19. Disucssion Papers Series. IZA DP No. 13411. IZA Institute for Labor Economics, disponible en http://ftp.iza.org/dp13411.pdf (Consultado el 15/12/2020).

Burki, T., 2020, “Covid-9 in Latin America”, en The Lancet, 20(5), 547-548, disponible en https://doi.org/10.1016/S1473-3099(20)30303-0

Campos-Nonato, I., Hernández-Barrera, L., Pedroza-Tobías, A., Medina, C., y Barquera, S., 2018, "Hipertensión arterial en adultos mexicanos: prevalencia, diagnóstico y tipo de tratamiento", en Salud Pública de México, 60(3), 233-243. Ensanut MC 2016, disponible en https://doi.org/10.21149/8813

Capolongo, S., Rebecchi, A., Buffoli, M., Appolloni, L., Signorelli, C., Fara, G. M., y D'Alessandro, D., 2020, "Covid-19 and Cities: from Urban Health strategies to the pandemic challenge. A Decalogue of Public Health opportunities", en Acta Bio-Medica: Atenei Parmensis, 91(2), 13-22, disponible en https://doi. org/10.23750/abm.v91i2.9615

Castañeda, C., y Ramos, G., 2020, "Principales pandemias en la historia de la humanidad", en Revista Cubana de Pediatría, 92(Supl. 1), e1183. Epub 20/07/2020, disponible en http://scielo.sld.cu/scielo.php?script=sci_arttext\&pi$\mathrm{d}=$ S0034-75312020000500014\&lng=es\&tlng=es

CEPAL, 15 de julio 2020, Comunicado de prensa. Comisión Económica para América Latina y el Caribe (CEPAL), 15 de julio 2020, disponible en https://www. cepal.org/es/comunicados/contraccion-la-actividad-economica-la-region-se-profundiza-causa-la-pandemia-caera-91 (Consultado el 10/12/2020).

DGE/SSA, 2021, Datos Abiertos. Covid-19. Dirección General de Epidemiología de la Secretaría de Salud de México (DGE/SSA), disponible en https://www.gob. $\mathrm{mx} /$ salud/documentos/datos-abiertos-152127 (Consultado el 16/12/2020).

Ejaz, H., Alsrhani, A., Zafar, A., Javed, H., Junaid, K., Abdalla, A. E., Abosalif, K., Ahmed, Z., y Younas, S., 2020, “Covid-19 and comorbidities: Deleterious 
impact on infected patients", en Journal of Infection and Public Health, 13(12), 1833-1839, disponible en https://doi.org/10.1016/j.jiph.2020.07.014

Gold, M. S., Sehayek, D., Gabrielli, S., Zhang, X., McCusker, C., y Ben-Shoshan, M., 2020, "Covid-19 and comorbidities: a systematic review and meta-analysis", en Postgraduate Medicine, 132(8), 749-755, disponible en https://doi.org/10.108 0/00325481.2020.1786964

González-Arellano, S., y Larralde Corona, A. H., 2019, "La forma urbana actual de las zonas metropolitanas en México: indicadores y dimensiones morfológicas", en Estudios Demográficos y Urbanos, 34(1), 11-42, disponible en http://dx.doi. org/10.24201/edu.v34i1.1799

Hernández-Garduño E., "2020, “Obesity is the comorbidity more strongly associated for Covid-19 in Mexico. A case-control study", en Obesity Research \& Clinical Practice, 14(4), 375-379, disponible en https://doi.org/10.1016/j. orcp.2020.06.001

Iacus, S.M., Santamaria, C., Sermi, F., Spyratos, S., Tarchi, D., y Vespe, M., 2020, "Human mobility and Covid-19 initial dynamics", en Nonlinear Dynamics, 101, 1901-1919, disponible en https://doi.org/10.1007/s11071-020-05854-6

INEGI, 2015, Encuesta Intercensal 2015. Instituto Nacional de Estadística y Geografía (INEGI), disponible en https://www.inegi.org.mx/programas/intercensal/2015/ (Consulta el 10/12/2020).

Kalabikhina, I., 2020, "Demographic and social issues of the pandemic. Population and Economics 4(2): 103-122, disponible en https://doi.org/10.3897/popecon.4.e53891

Larrea, F., 2007, “La viruela: ¿ha muerto?”, en Revista del Instituto Nacional de Higiene Rafael Rangel, 38(1), 34-38, disponible en http://ve.scielo.org/scielo. php?script=sci_arttext\&pid=S0798-04772007000100006\&lng=es\&tlng=es.

Lee, V. J., Ho, M., Kai, C. W., Aguilera, X., Heymann, D., y Wilder-Smith, A., 2020, "Epidemic preparedness in urban settings: new challenges and opportunities", en The Lancet. Infectious diseases, 20(5), 527-529, disponible en https:// doi.org/10.1016/S1473-3099(20)30249-8

Moreno-Sánchez, F., Coss Rovirosa, M. F., Alonso de León, M. T., y Elizondo Ochoa, A., 2018, "Las grandes epidemias que cambiaron el mundo", en Anales Médicos de la Asociación Médica del Centro ABC, 63(2), 151-156, disponible en https://www.medigraphic.com/pdfs/abc/bc-2018/bc182p.pdf

Mueller, A., McNamara, M., y Sinclair, D, 2020, "Why does Covid-19 disproportionately affect older people?", in Aging, 12(10), 9959-9981, disponible en https:// doi.org/10.18632/aging.103344

OMS, 2020, Policy Brief: COVID-19 in an Urban World. Organización Mundial de la Salud (OMS), disponible en https://unsdg.un.org/es/resources/informe-de-politicas-Covid-19-en-un-mundo-de-poblacion-urbana (Consultado el 11/01/2021). 
OMS, 2021, WHO Coronavirus Disease (Covid-19) Dashboard. Organización Mundial de la Salud (OMS), disponible en https://covid19.who.int/ (Consultado el 11/01/2021)

ONU, 2018, The World's cities in 2018: Data booklet. Organización de las Naciones Unidas (ONU). New York.

OPS, 2020, Actualización epidemiológica enfermedad por coronavirus (Covid-19). Organización Panamericana de la Salud (OPS), disponible en https://www.paho. org/es/file/71105/download?token=t3ekzUeP (Consultado el 27/12/2020)

Parra-Bracamonte, G. M., Lopez-Villalobos, N., y Parra-Bracamonte, F. E, 2020, "Clinical characteristics and risk factors for mortality of patients with Covid-19 in a large data set from Mexico", en Annals of Epidemiology, 52, 93-98, disponible en https://doi.org/10.1016/j.annepidem.2020.08.005

Pradilla, E., 2016, "Zona Metropolitana del Valle de México: neoliberalismo y contradicciones urbanas", en Sociologias, Porto Alegre, 18(42), 54-89, disponible en https://doi.org/10.1590/15174522-018004203.

QGIS.org, 2021, QGIS Geographic Information System. QGIS Association, http:// www.qgis.org

Ramos, M., 2020, Coronavirus: ¿por qué América Latina es la región con más muertes en el mundo? BBC News. 19 de octubre 2020, disponible en https://www. bbc.com/mundo/noticias-america-latina-54597871 (consultado el 10/12/2020).

Reyes, R., Ahn, R., Thurber, K., y Burke, T. F., 2013), “Urbanization and Infectious Diseases: General Principles, Historical Perspectives, and Contemporary Challenges", in Fong, I. W. (editor). Challenges in Infectious Diseases. New York: Springer.

SEDATU, CONAPO, INEGI) 2018, Delimitación de las zonas metropolitanas de México 2015. Secretaría de Desarrollo Agrario, Territorial y Urbano (SEDATU) / Consejo Nacional de Población (CONAPO) / Instituto Nacional de Estadística y Geografía (INEGI), disponible en https://www.gob.mx/sedatu/documentos/ delimitacion-de-las-zonas-metropolitanas-de-mexico-2015-149891 (consulta 10/12/2020).

Sharifi, A., y Khavarian-Garmsir, A. R., 2020, “The Covid-19 pandemic: Impacts on cities and major lessons for urban planning, design, and management", en Science of the Total Environment, 749, 1-2, disponible en https://doi.org/10.1016/j. scitotenv.2020.142391

Shoals, J., 2018, Epidemics and Pandemics. Pennsylvania: Mason Crest.

Singer M., 2020, "Deadly Companions: Covid-19 and Diabetes in Mexico", en Medical Anthropology", 39(8), 660-665, disponible en https://doi.org/10.1080/01 459740.2020.1805742

Sobrino, L. J., 2011, “La urbanización en el México contemporáneo", en Notas de Población, 94, 93-123, disponible en https://repositorio.cepal.org/bitstream/handle/11362/12898/np94093122_es.pdf?sequence=1\&isAllowed=y 
Suárez, V., Suarez Quezada, M., Oros Ruiz, S., y Ronquillo de Jesús, E., 2020, "Epidemiology of Covid-19 in Mexico: from the 27th of February to the 30th of April 2020. Epidemiología de Covid-19 en México: del 27 de febrero al 30 de abril de 2020", en Revista Clínica Española, 220(8), 463-471, disponible en https://doi. org/10.1016/j.rce.2020.05.007

Suárez Lastra, M., Váldes González, C. M., Galindo Pérez, M. C., Salvador Guzmán, L. E., Ruíz-Rivera, N., Alcántara-Ayala, I... Garnica-Peña, R., 2020, "Índice de vulnerabilidad ante el COVID-19 en México", en Investigaciones Geográficas, (publicación electrónica), 1-22. doi: 10.14350/rig.60140

Trejo Nieto, A., 2013, "Las economías de las zonas metropolitanas de México en los albores del siglo XXI”, en Estudios Demográficos y Urbanos, 28(3), 545-591, disponible en https://estudiosdemograficosyurbanos.colmex.mx/index.php/edu/ article/view/1447/1440

Yang, W., Wang, X., Zhang, K., y Ke, Z., 2020, “Covid-19, Urbanization pattern and economic recovery: an analysis of Hubei, China", in International Journal of Environmental Research and Public Health, 17(24), 9577, disponible en https:// doi.org/10.3390/ijerph17249577

Zhu, N., Zhang, D., Wang, W., Li, X., Yang, B., Song, J... for the China Novel Coronavirus Investigating and Research Team, 2020, "A Novel Coronavirus from Patients with Pneumonia in China, 2019", in The New England Journal of Medicine, 382, 727-733. DOI: 10.1056/NEJMoa2001017

\section{RESUMEN CURRICULAR DE LOS AUTORES}

\section{Daniel Lozano Keymolen}

Es doctor en Estudios de Población por El Colegio de México. Se desempeña como profesor e investigador de tiempo completo en el Centro de Investigación y Estudios Avanzados de la Población de la Universidad Autónoma del Estado de México. Ha publicado diversos artículos de investigación sobre el perfil de la salud y acerca de los procesos de desigualdad en el estado de salud de las personas en edades avanzadas. Pertenece al Sistema Nacional de investigadores Nivel I de Conacyt de México.

Dirección electrónica: dlozano@colmex.mx

\section{Sergio Cuauhtémoc Gaxiola Robles Linares}

Actuario por la Facultad de Ciencias de la Universidad Nacional Autónoma de México. Maestro en Estudios de Población por la Facultad Latinoamericana de Ciencias Sociales (sede México). Doctor en Estudios de Población por El Colegio de México. Profesor de Tiempo Completo del Centro de Investigación y Estudios Avanzados de la Población de la Universidad Autónoma del Estado de México (UAEM) y miembro del Sistema Nacional de Investigadores (SNI). Docente en programas de licenciatura 
de la UAEM. Ha publicado varios artículos. Líneas de investigación: envejecimiento, mercados de trabajo y pobreza.

Dirección electrónica: srobles99@gmail.com

Bernardino Jaciel Montoya Arce

Es Licenciado, Maestro y Doctor en Sociología por la Universidad Nacional Autónoma de México (UNAM). Pertenece al Sistema Nacional de Investigadores Nivel I, cuenta con Perfil Deseable PROMEP. Actualmente es Coordinador del Centro de Investigación y Estudios Avanzados de la Población de la Universidad Autónoma del Estado de México (CIEAP-UAEM). Entre sus publicaciones recientes se encuentran Demografia indígena en el Estado de México (coautor), 2013; Análisis demográfico del envejecimiento en el Estado de México (compilador), UAEM, 2011 y "La educación indígena en el Estado de México", en Papeles de Población, 2013.

Dirección electrónica:bjmontoyaa@uaemex.mx 